\title{
CAUSALIDAD ENTRE LAS IMPORTACIONES Y EL CRECIMIENTO ECONÓMICO: EVIDENCIA EMPÍRICA PARA EL DEPARTAMENTO DEL CAUCA (COLOMBIA)*
}

\author{
ANDRÉS MAURICIO GÓMEZ SÁNCHEZ** \& ZORAIDA RAMÍREZ GUTIÉRREZ**** \\ UNIVERSIDAD DEL CAUCA
}

Recibido/ Received/ Recebido: 07/03/2016 - Aceptado/ Accepted / Aprovado: 30/09/2016

\begin{abstract}
Resumen
Este documento investiga si el crecimiento económico en el departamento del Cauca determina el incremento de sus importaciones o si por el contrario son las importaciones las que han impulsado el PIB en el periodo 1960-2013. Para lograr este objetivo, se recurre a un modelo SVAR con su previo análisis de cointegración. Se implementa también una función impulso-respuesta para reforzar los hallazgos. Los resultados sugieren que el PIB per cápita del Cauca contemporáneo incide en las importaciones futuras y no viceversa, lo que implica, por ejemplo, que los tratados de libre comercio no son suficientes para impulsar dichas importaciones en el largo plazo.
\end{abstract}

Palabras clave: Importaciones; PIB; Crecimiento Económico; Modelos VAR; Colombia.

\section{CAUSALITY BETWEEN IMPORTS AND ECONOMIC GROWTH: EMPIRICAL EVIDENCE FOR THE CAUCA DEPARTMENT (COLOMBIA)}

\begin{abstract}
This paper investigates if the Cauca's department economic growth determines the increase of its imports or if on the contrary it is the imports that have boosted the GDP in the period 19602013. To achieve this objective, an SVAR model is used with its previous cointegration analysis. A boost-response function is also implemented to reinforce the findings. The results suggest that the contemporary Cauca's per capita GDP affects future imports and not vice versa, which implies for example that free trade agreements are not enough to boost these imports in the long term. This
\end{abstract}

Artículo de investigación derivado del proyecto: "Eficiencia del Transporte Público en la Ciudad de Morelia, Michoacán en el año 2015 a través del Análisis de la Envolvente de Datos"

* Docente, Departamento de Ciencias Económicas, Universidad del Cauca, Popayán, Colombia. Miembro del Grupo de investigación Entropía, Universidad del Cauca. Economista, Universidad del Valle, Cali, Colombia. Especialista, Gerencia de Proyectos, Universidad del Cauca, Popayán, Colombia. Magíster en Economía Aplicada, Universidad del Valle, Cali, Colombia. Estudiante de Doctorado en Economía Industrial, Universidad de Valencia, España. Dirección: Dirección: Carrera 2 con calle 15, Edificio Sector Tulcán, Pomona, Universidad del Cauca, Popayán. Correo electrónico: amgomez@unicauca.edu.co.

*** Docente, Departamento de Ciencias Contables, Universidad del Cauca, Popayán, Colombia. Coordinadora del Grupo de Estudio e Investigación en Finanzas y Gestión (GREIFG), Universidad del Cauca. Contadora Pública, Universidad del Quindío, Armenia, Colombia. Especialista en Administración Financiera, Universidad Gran Colombia, Armenia, Colombia. Magíster en Administración Económica y Financiera, Universidad Tecnológica de Pereira, Pereira, Colombia. Magíster en Administración de Empresas con énfasis en Finanzas Corporativas, Universidad Viña del Mar, Chile. Estudiante de Doctorado en Contabilidad y Finanzas Corporativas, Universidad de Valencia, España. Dirección: Carrera 2 con calle 15, Edificio Sector Tulcán, Pomona, Universidad del Cauca, Popayán. Correo electrónico: zramirez@unicauca.edu.co. 
paper investigates if the economic growth in the department of Cauca determines the increase of its imports or if, on the contrary, it is the imports that have boosted the GDP in the period 19602013. To achieve this objective, an SVAR model is used with its previous cointegration analysis. A boost-response function is also implemented to reinforce the findings. The results suggest that the per capita GDP of the contemporary Cauca affects future imports and not vice versa, which implies, for example, that free trade agreements are not enough to boost these imports in the long term.

Keywords: Imports; GDP; Economic growth; VAR models; Colombia.

\title{
CAUSALIDADE ENTRE AS IMPORTAÇÕES E O CRESCIMENTO ECONÔMICO: EVIDÊNCIA EMPÍRICA PARA O DEPARTAMENTO DO CAUCA (COLÔMBIA)
}

\begin{abstract}
Resumo
Este documento pesquisa se o crescimento econômico no departamento do Cauca determina o incremento de suas importações ou se pelo contrário são as importações as que têm impulsionado o PIB no período 1960-2013. Para conseguir este objetivo, recorre-se ao modelo SVAR com sua prévia análise de cointegração. Implementa-se também uma função impulso-resposta para reforçar os resultados. Estes sugerem que o PIB per capita do Cauca contemporâneo incide nas importações futuras e não o contrário, o que implica, por exemplo, que os tratados de livre comércio não são suficientes para impulsionar estas importações a longo prazo.
\end{abstract}

Palavras chave: Importações; PIB; Crescimento Econômico; Modelos VAR ; Colômbia.

Gómez, A. \& Ramírez, Z. (2017) Causalidad entre las importaciones y el crecimiento económico: evidencia empírica para el departamento del Cauca (Colombia). En: Revista de la Facultad de Ciencias Económica: Investigación y Reflexión. rev.fac.cienc.econ, XXV (2), DOI: https://doi.org/10.18359/rfce.3068

JEL: B22, C01, F19, R11.

\section{Introducción}

Desde la aparición de los mercantilistas en el siglo XVI, pasando por David Ricardo (1817-1973) y Keynes (1936) al igual que por Bahmani-Oskooee \& Gobinda (2004), hasta Krugman \& Obsfield (2006), se ha expuesto la importancia del comercio internacional para el crecimiento y desarrollo económico de los países. Es bien sabido que las exportaciones elevan los niveles de ingreso, redundando en una mayor capacidad de compra de bienes nacionales y/o foráneos, por parte de los demandantes internos; y del lado de las importaciones la consecución de bienes y servicios, materias primas o insumos de producción incrementan la utilidad en los consumidores y permiten ampliar las posibilidades de producción en las empresas.
En este sentido, los estudios contemporáneos se han centrado en indagar acerca del rol de las exportaciones en el crecimiento económico, dejando a un lado a las importaciones y su papel en dicho crecimiento. ${ }^{1}$ Esto obedece a la continuidad de esa idea tradicional de los mercantilistas que justifica el aumento de la riqueza (o puesto en términos actuales, el crecimiento) elevando las exportaciones y minimizando las importaciones, es decir manteniendo una balanza comercial siempre favorable. Bajo esta premisa, un conjunto de economistas han abierto el debate alrededor de la relación de causalidad entre el PIB y las exportaciones tales como Panas \& Vamvoukas (2002), Balaguer \& Cantavella-Jordá (2001), Abdulai \& Jaquet (2002), Awokuse (2003),

1 El crecimiento económico en este documento hace referencia a variaciones en el PIB per cápita y no del PIB total. 
Sharma \& Panagiotidis, (2005) y Jin (2002), Alonso \& Patiño (2007), entre muchas otras.

Dentro de ellos, una corriente apoya la hipótesis "Exports-driven growth" (EDG): el aumento en las exportaciones genera un incremento en la demanda externa ceteris paribus, fortaleciendo la demanda agregada y por tanto el nivel de actividad económica, al igual que los ingresos provenientes de dicha actividad, y se traduce finalmente en crecimiento económico. La otra corriente afirma que la situación es la contraria, es decir se defiende la hipótesis de "Growth-led exports" (GLE): son los mayores niveles de actividad económica los impulsores o conductores de las exportaciones, ya que la producción dirigida al exterior solo puede concebirse en un escenario de mayor crecimiento económico, debido a su generación de producciones excedentarias que pueden ser comercializadas fuera del país. Los resultados empíricos muestran que para algunas regiones existe un tipo de causalidad y para otras la causalidad es contraria.

Del lado de las importaciones la dinámica no ha sido relevante en este tipo de discusiones y modelaciones, porque el interés se ha centrado en la balanza comercial teniendo a la variable exportaciones como conductora del superávit y en consecuencia del crecimiento económico. En efecto, sí año tras año las importaciones superan a las exportaciones se hablará de un déficit comercial y de manera contraria la economía presentará un superávit.

A pesar de ello, es importante denotar que las importaciones pueden incidir en el comportamiento del PIB de una nación en dos vías: primero, en el corto plazo pueden resultar lesivas para el crecimiento del PIB, porque al aumentar las importaciones se perjudica o lesiona la demanda externa. Algunos autores como los de la escuela Cepalina en cabeza de Prebisch (1950) con el modelo de Industrialización por Sustitución de Importaciones o modelo "ISI", aplicado en América Latina, apoyaban la idea de que las importaciones afectaban de forma lesiva al PIB. Esta propuesta está en consonancia con la denominada "Ley de Thirlwall" (Thirlwall, 1979), la cual en términos generales propone que el incremento de las exportaciones mejora el creci- miento económico, mientras que el incremento de las importaciones lo empeora.

En segundo lugar, a largo plazo las importaciones pueden jugar un papel fundamental en el crecimiento económico de los países, pues estas generalmente no se hacen por bienes finales sino por bienes intermedios, siendo por tanto relevante los tipos de bienes importados y no el valor de su monto anual. Autores como Griswold (2010), Li, Greenaway \& Hine (2003) respaldan esta última idea. Así, algunos autores consideran la relación y efectos importaciones - crecimiento económico, aunque su comportamiento no es igual en el corto que en largo plazo.

De otro lado, autores como Khan (1975), Khan \& Ross (1977) y Bahmani-Oskooee \& Gobinda (2004), se basan en las funciones de demanda marshallianas; donde la importación de bienes y servicios foráneos de una nación o región, como cualquier demanda de este tipo, depende entre otras cosas del ingreso, es decir del PIB. Así, estos autores apoyan la idea general de la relación entre los niveles de ingreso, las tasas de crecimiento del PIB, y la mayor disponibilidad a pagar por bienes extranjeros, fenómeno determinante del aumento de las importaciones en el mediano y largo plazo. En este caso es entonces el PIB quien determina el nivel de importaciones futuras de un país o región y no viceversa. Inclusive el mismo modelo keynesiano, a través de su función de importación, plantea su dependencia del PIB.

Bajo este panorama, se abre otro debate ya que tampoco es clara la relación de causalidad entre las importaciones y el crecimiento económico. Por tal razón en este documento, a la luz de los estudios sobre exportaciones, plantea la existencia de la hipótesis "Growth-driven imports" (GDI) en las economías; es decir que el crecimiento económico es quien impulsa a las importaciones, o de manera contraria, se plantea la hipótesis "Imports-led growth" (ILG), donde son las importaciones las responsables de elevar el PIB.

Dichas relaciones de causalidad entre las importaciones y el crecimiento económico no se han explorado empíricamente, ni a nivel macroeconómico 
ni a nivel regional en Colombia. Recientemente, en el departamento del Cauca las importaciones han superado a las exportaciones. Para el año 2012 se registró en términos reales una cifra cercana a los US\$384 millones a precios CIF contra un total de US\$63 millones a precios FOB, mostrando una balanza comercial deficitaria, pero se observa que el PIB en ese mismo periodo ha experimentado un crecimiento positivo de $7,6 \%$, situación recurrente en el Cauca desde comienzos de este milenio. El estudio de dichas causalidades cobra aún más importancia en un mundo globalizado, de cara a los tratados comerciales firmados en nuestro país, y aquellos que se encuentran en proceso, los cuales afectan directamente a las economías de las regiones, dando luces sobre la pertinencia o no de dichos convenios en materia de crecimiento económico.

En este orden de consideraciones, esta investigación tiene por objetivo esclarecer de forma empírica si las importaciones impulsan el crecimiento económico (hipótesis GDI) o viceversa (hipótesis ILG) en el departamento del Cauca. Para logarlo, se implementa un análisis de cointegración y un modelo SVAR con funciones impulso respuesta que permitan simular los comportamientos futuros en el corto y mediano plazo de las importaciones y el PIB departamental. Este documento se divide en cuatro apartados después de la respectiva introducción. La primera parte plantea el marco teórico y de estudios aplicados en esta materia, la segunda muestra un panorama de las importaciones en el Cauca; el tercer apartado implementa la modelación econométrica para finalmente esbozar algunas conclusiones, reflexiones y caminos de futuras investigaciones.

\section{Referentes Teóricos y Estudios Aplicados}

Las importaciones definidas como todos los bienes y servicios adquiridos por un país o región del extranjero para distribuirlos en su interior, no ha tenido una única mirada en la economía pues a nivel teórico y práctico presenta diversos efectos en el crecimiento económico. En general, las importaciones permiten adquirir bienes y servicios que no se producen al interior del país lo cual beneficia a consumidores en precios, calidad o variedad y a las empresas en la consecución de materiales, insumos de producción o mejores tecnologías; pero de otro lado genera competencia a la industria ya establecida o naciente en país importador y lesiona el mercado laboral interno, ya que genera desempleo.

Históricamente hablando, el papel del comercio internacional y de las importaciones en la economía data de la época del mercantilismo (1500-1750); su génesis se dio en las monarquías de la época con el fin de promover la acumulación de las naciones y fortalecer el Estado-Nación a través del sostenimiento de unas exportaciones mayores que las importaciones, es decir mantener una balanza comercial favorable para incrementar la riqueza nacional, en el marco de un fuerte proteccionismo e intervencionismo estatal.

En el siglo siguiente y con una visión totalmente diferente, David Ricardo (1817-1973), consideraba a las importaciones jugando un papel preponderante en la economía inglesa, ya que la liberación de las importaciones de cereales evitaba el estado estacionario, pues de otra forma se generaban aumentos en la renta de la tierra, generando descensos en la tasa de beneficios y afectando el progreso económico. Este análisis dio origen a conceptos fundamentales para la economía ortodoxa como el de rendimientos marginales decrecientes y el de ventaja comparativa, en oposición a las ventajas absolutas de Smith (1776-1987). Estos elementos fueron tomados posteriormente por el modelo de Heckscher-Ohlin, promulgando que el proceso de exportación de los países debe especializarse en la producción con costos de oportunidad bajos. La producción intensiva debe basarse en factores de producción abundantes, y de forma contraria, se deben importar bienes cuyo costo de oportunidad interno sea muy elevado, justificado en la escases de recursos.

Tres años más tarde, el modelo macroeconómico keynesiano introduce el concepto de exportaciones netas (exportaciones menos importaciones) dentro de la cual existe la función de importación. Esta depende de los niveles de ingreso, y su pendiente es la propensión marginal a importar. De acuerdo con esta ecuación, las exportaciones netas dependen inversamente del ingreso, es decir si aumen- 
ta el ingreso se eleva la capacidad de compra por bienes extranjeros cayendo las exportaciones netas, ceteris paribus y viceversa. De aquí se desprende una demarcada conclusión, ya que las importaciones al entrar de forma negativa en la balanza comercial implican que un aumento de ellas reduce el PIB de los países, es decir, mayores niveles de importación frenan el crecimiento económico. Vale la pena mencionar que la relación también puede ser contraría, es decir, altas importaciones frenan el consumo porque hay menos ingreso y esto frena el gasto agregado y también el crecimiento. Las importaciones dependen del ingreso, y el ingreso también depende de las importaciones.

En la época de la postguerra, en el contexto latinoamericano se denota la importancia de crear un modelo económico para llevar a los países emergentes por el camino del desarrollo y/o crecimiento económico. El modelo se denomina Modelo de Industrialización por Sustitución de Importaciones o modelo ISI, propuesto por Prebisch (1950), y complementado por Furtado (1969), Pinto (1970) y Sunkel \& Paz (1970). Este modelo se basa en la premisa de considerar a América Latina sin autonomía relativa a su desarrollo económico y dependiente de acontecimientos y políticas de los países desarrollados, es decir, la periferia (países emergentes) depende económicamente del centro (países desarrollados), situación inhibitoria de su crecimiento económico. La idea entonces para revertir el proceso es dejar de importar bienes y servicios del extranjero e impulsar la producción de ellos al interior del país, mostrando una balanza comercial favorable y por tanto un mayor impulso a la industria nacional, generándose una menor dependencia de los mercados mundiales. Argumentos similares al modelo mercantilista, con diferencias relativas a este último, por propender hacia la acumulación de oro y riquezas más no hacia el desarrollo de un país como tal, pues dicho concepto aún no se conocía. La conclusión del modelo, siguiendo a Ramales (2008), es que el comercio internacional lejos de beneficiar a los países en desarrollo los perjudica, afirmación contraria a los planteamientos y hallazgos de Krugman \& Obsfield (2006).
En la década del setenta, una vez el modelo ISI estaba perdiendo vigencia, Khan (1975) construye un modelo económico donde como cualquier función de demanda marshalliana depende básicamente del ingreso y de los precios, es decir, plantea un modelo de demanda de importaciones que es función del PIB real y del tipo de cambio real, incluyendo un shock de demanda a través de una variable dicotómica. En este caso, un mayor ingreso eleva las importaciones y no viceversa. Luego de los años ochenta se destacan otros modelos de demanda de importaciones como los de Metwally \& Abdel-Rhman (1985), y el de Asseery \& Perdkikis (1993), quienes introducen algún grado de dinámica al construir un modelo basado en Khan (1975) pero con la inclusión de un rezago en las importaciones.

Más recientemente, algunos autores como Eaton \& Kortum (1999); Keller (2002); Li, Greenway \& Hane (2003) apoyan la visión que el comercio internacional permite el crecimiento económico debido a que las importaciones impulsan la difusión de nuevas y mejores tecnologías hacia países donde no es posible desarrollarlas, y como ella es clave para explicar las diferencias en el ingreso y la productividad de los países, su esparcimiento permite que las brechas entre países no sea tan elevada, ceteris paribus. Estos aportes están asociados a la hipótesis de catching up, la cual sostiene en términos generales que los países emergentes o pobres pueden elevar la productividad total de los factores (PTF) a una tasa más rápida que la de los países desarrollados debido a que es más barato imitar las tecnología que crearlas (Maudos, Pastor \& Serrano, 1998).

En este mismo sentido, Markusen \& Rutherford (2002) encuentran que una importante fuente de transmisión del conocimiento de los países desarrollados hacia los emergentes se logra a través de la importación de servicios de expertos tales como ingenieros y consultores de gestión, ya que la incorporación de su conocimiento a los procesos productivos por una lado sería menos costoso que si se hiciese con personal del interior, y por otro, se generan efectos spillover de los expertos hacia los trabajadores poco calificados, elevando sus niveles de productividad en el mediano y largo plazo. Siguiendo a Becker 
(1964) y Mincer (1974), estos conceptos se formalizan a través de la transferencia tecnológica mediante el desarrollo y transferencia del capital humano.

Otros autores consideran que las importaciones ayudan al crecimiento económico a través de la exportación de turismo. En efecto, Nowak, Sahli \& Cortés (2007) a través de un sofisticado modelo matemático y Brida, Pereyra, Such \& Pulina (2011), plantean que el crecimiento económico sostenido se logra mediante la importación de capitales extranjeros en su totalidad financiados a través del turismo receptor. Es decir, el país exportador del turismo debe previamente importar bienes indus- triales, maquinaria y tecnología para impulsar nueva infraestructura y capital humano, lo cual genera mayores niveles de crecimiento en la nación.

En el caso de los estudios aplicados, desde el lado de las importaciones y su causalidad hacia el PIB se encuentran pocos estudios, pues generalmente se encuentran aplicaciones para el caso de las exportaciones y si estas cumplen con la hipótesis de "Growth-driven Exports" (GDE) o por el contrario la "Exports-led growth" (ELG), es decir si las exportaciones impulsan el crecimiento o viceversa. A continuación se enlistan algunos de los estudios aplicados más recientes:

Tabla 1. Resumen Estudios Empíricos para Hipótesis GDE, ELG, GDI e ILG

\begin{tabular}{|c|c|c|c|c|}
\hline Autor(es) & Año & Objetivo & Metodología & Resultados \\
\hline Khan & 1975 & $\begin{array}{l}\text { Indagar por la relación estática } \\
\text { entre las importaciones y el } \\
\text { ingreso }\end{array}$ & $\begin{array}{l}\text { Estimación por } \\
\text { MCO Demanda } \\
\text { Marshalliana }\end{array}$ & Un mayor ingreso eleva las importaciones y no viceversa \\
\hline Metwally \& Abdel-Rhman & 1985 & $\begin{array}{l}\text { Indagar por la relación dinámi- } \\
\text { ca entre las importaciones y el } \\
\text { ingreso }\end{array}$ & $\begin{array}{l}\text { Estimación por } \\
\text { MCO Demanda } \\
\text { Marshalliana }\end{array}$ & $\begin{array}{l}\text { Mayores importaciones rezagadas en el tiempo elevan las } \\
\text { importaciones contemporáneas }\end{array}$ \\
\hline $\begin{array}{l}\text { Balaguer \& Cantavella- } \\
\text { Jordá }\end{array}$ & 2002 & $\begin{array}{l}\text { Examinan la relación entre los } \\
\text { ingresos reales y las exporta- } \\
\text { ciones reales en España }\end{array}$ & Modelo VAR & $\begin{array}{l}\text { El crecimiento es impulsado por las exportaciones en } \\
\text { condiciones de libre comercio, pero cuando se considera } \\
\text { el siglo en su conjunto, existe una causalidad unidireccio- } \\
\text { nal que va desde el ingreso hacia las exportaciones }\end{array}$ \\
\hline Awokuse & 2003 & $\begin{array}{l}\text { Examinar la hipótesis ELG en } \\
\text { Canadá. }\end{array}$ & $\begin{array}{l}\text { Modelos VCE y } \\
\text { VAR }\end{array}$ & $\begin{array}{l}\text { La causalidad a la Granger es unidireccional y va desde } \\
\text { las exportaciones reales hacia el PIB real. }\end{array}$ \\
\hline Panas \& Vamvoukas & 2002 & $\begin{array}{l}\text { Explorar la causalidad entre las } \\
\text { exportaciones y el crecimiento } \\
\text { del producto en la economía } \\
\text { griega. }\end{array}$ & $\begin{array}{l}\text { Modelos VCE y } \\
\text { VAR multivariados }\end{array}$ & $\begin{array}{l}\text { La hipótesis GDE es válida, existe una relación causal } \\
\text { fuerte y consistente de crecimiento de la producción ha- } \\
\text { cia las exportaciones en el largo plazo }\end{array}$ \\
\hline Jin & 2002 & $\begin{array}{l}\text { Validar la hipótesis GDE en } \\
\text { Corea }\end{array}$ & Modelos VAR & $\begin{array}{l}\text { El crecimiento impulsa las exportaciones y no al contra- } \\
\text { rio. }\end{array}$ \\
\hline Alonso \& Patiño & 2007 & $\begin{array}{l}\text { Investigar la validez de la hi- } \\
\text { pótesis EDG para el Valle del } \\
\text { Cauca }\end{array}$ & $\begin{array}{l}\text { Modelos VAR con } \\
\text { funciones Impulso- } \\
\text { Respuesta }\end{array}$ & $\begin{array}{l}\text { Se valida la relación causal del crecimiento económico } \\
\text { basado en exportaciones. }\end{array}$ \\
\hline Nowak, Sahli \& Cortés & 2007 & $\begin{array}{l}\text { Validar la hipótesis TKIG } \\
\text { (tourism-capital good imports- } \\
\text { growth). }\end{array}$ & Modelos VAR & $\begin{array}{l}\text { Existe causalidad entre el turismo, la importación de bie- } \\
\text { nes de capital y el crecimiento económico }\end{array}$ \\
\hline Kee, Nicita \& Olarreaga & 2008 & $\begin{array}{l}\text { elasticidades de la demanda de } \\
\text { importaciones para un amplio } \\
\text { grupo de países a nivel desa- } \\
\text { gregado }\end{array}$ & $\begin{array}{l}\text { Estimación deman- } \\
\text { da de importacio- } \\
\text { nes a través de una } \\
\text { función translog. }\end{array}$ & \\
\hline Gómez \& Salazar & 2014 & $\begin{array}{l}\text { Indagar por los determinantes } \\
\text { a largo plazo de las importacio- } \\
\text { nes para los departamentos de } \\
\text { la costa Pacífica colombiana }\end{array}$ & $\begin{array}{l}\text { Demanda a la Khan } \\
\text { con modelos MCE }\end{array}$ & $\begin{array}{l}\text { Existe una relación estable a largo plazo entre las impor- } \\
\text { taciones totales, la tasa de cambio y el PIB. }\end{array}$ \\
\hline
\end{tabular}

Fuente: Elaboración propia. 
Las estimaciones de la relación de causalidad se basan en modelos VEC y VAR estándar, y posiblemente esta sea la causa de que los resultados sean disimiles. Por tanto se propone en este estudio formular un modelo VAR estructural (SVAR) para capturar con aportes teóricos una relación más acertada de la causalidad.

\section{Las Importaciones en el Cauca}

Históricamente el comportamiento de las importaciones del Cauca tiene un antes y un después a partir del año 1992. Como es previsible, este punto de quiebre se origina por la apertura económica por la que atravesaba el país a principios de la década del noventa, en el gobierno de César Gaviria.

Gráfico 1. Importaciones y PIB per cápita del Cauca Normalizados.

Miles de Millones de Pesos (2005=100). 1960-2013.

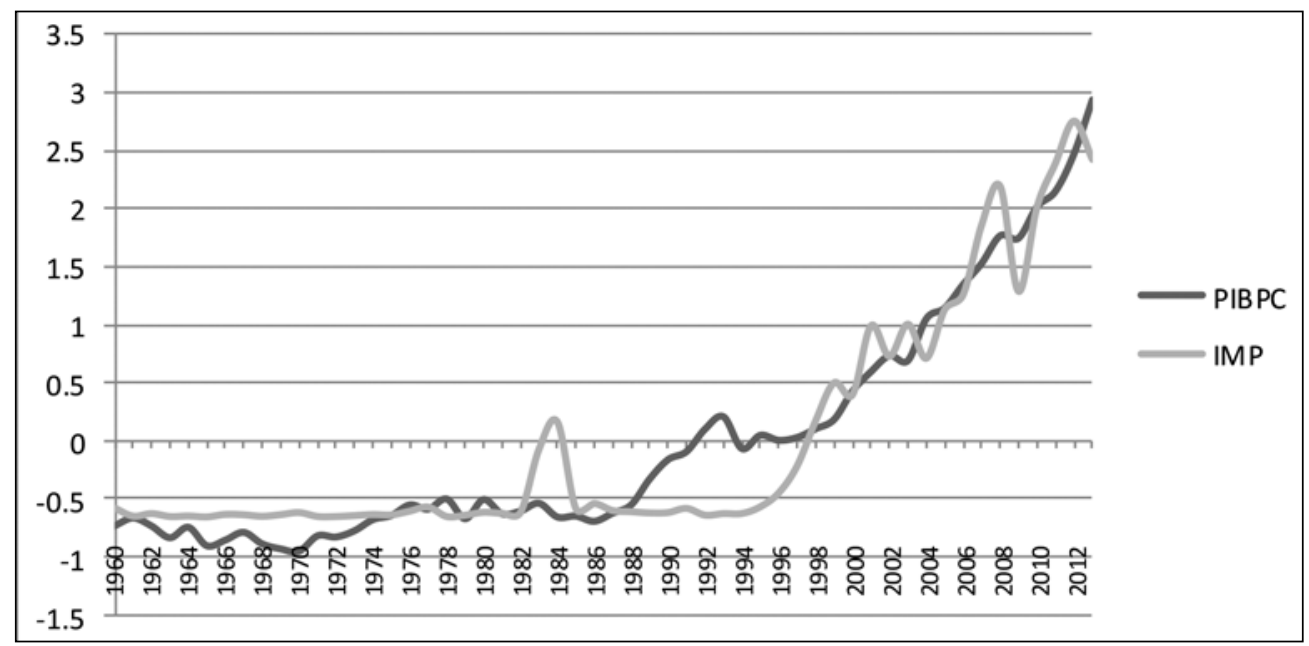

Fuente: Elaboración propia con datos DANE y SITEC.

PIBPC: PIB per cápita del Cauca, IMP: Importaciones Cauca.

Por ser cifras normalizadas, el eje Y debe ser entendido como desviaciones estándar.

En efecto, desde 1960 hasta 1991, las importaciones del departamento en promedio ascendieron tan solo a 18.3 millones de dólares, aunque desde 1992 hasta 2013, alcanzaron 358.3 millones de dólares en promedio. Para la última década analizada, las importaciones evidencian un comportamiento poco estable debido a incertidumbre en los mercados generada por la crisis económica y financiera mundial, sin embargo, siguiendo al informe del Perfil del Comercio Exterior del Cauca realizado por la Cámara de Comercio del Cauca (2013), el coeficiente de penetración de importaciones, es decir la proporción del mercado doméstico que se abastece con importaciones en un periodo, fue del $9.9 \%$ en 2010 y del $10 \%$ en 2011 . Una excepción a lo anterior, como se evidencia en el gráfico 1 , fue el repunte bastante fuerte en las importaciones en los años 1983 y 1984, debido al terremoto acaecido en Popayán, situación que obligó a la importación de diversos insumos de construcción para la restauración de la ciudad y de otros servicios (Cardona, et al., 2004, 22).

De otro lado, en el corto plazo se encuentra que antes del año 1992, la relación entre el ciclo de las importaciones frente al del ciclo de la economía departamental se comporta de manera procíclica de manera contemporánea, es decir que si el PIB 
per cápita (o las importaciones) se elevan en un determinado periodo, las importaciones (o el PIB per cápita) se elevan en el mismo periodo, aunque su relación no es demasiado fuerte ya que su correlación solo es cercana a 0,05. Una posible explicación obedece a los bajos niveles mostrados por las importaciones en ese periodo (ver gráfico 1) lo que posibilita una balanza comercial favorable que se refleja en el PIB per cápita departamental.

Ya para los años 1993-2013 la relación es un poco diferente, pues en primera instancia los ciclos se vuelven menos volátiles de forma contemporánea, y aunque su relación sigue siendo procíclica, ésta es más fuerte. En otras palabras, las expansiones (o contracciones) del PIB per cápita generan incrementos (o contracciones) en las importaciones y viceversa, para el mismo periodo, siendo dichas variaciones conjuntas más moderadas, pero su relación más fuerte pues su correlación es de 0.36. Como los valores de las correlaciones no muestran causalidad, no se sabe si a priori las importaciones causan al PIB per cápita o viceversa y menos si lo hacen de manera contemporánea, adelantada o rezagada, por esta razón la implementación de un modelo SVAR se hace necesaria para poder esclarecer dicha relación de manera dinámica. Posiblemente las mayores importaciones mostradas después de 1992 tengan relación con este comportamiento vía balanza comercial

Gráfico 2. Ciclo de las Importaciones y PIB per cápita del Cauca Normalizados.

Miles de Millones de Pesos (2005=100). Filtro de Kalman. 1960-2013.

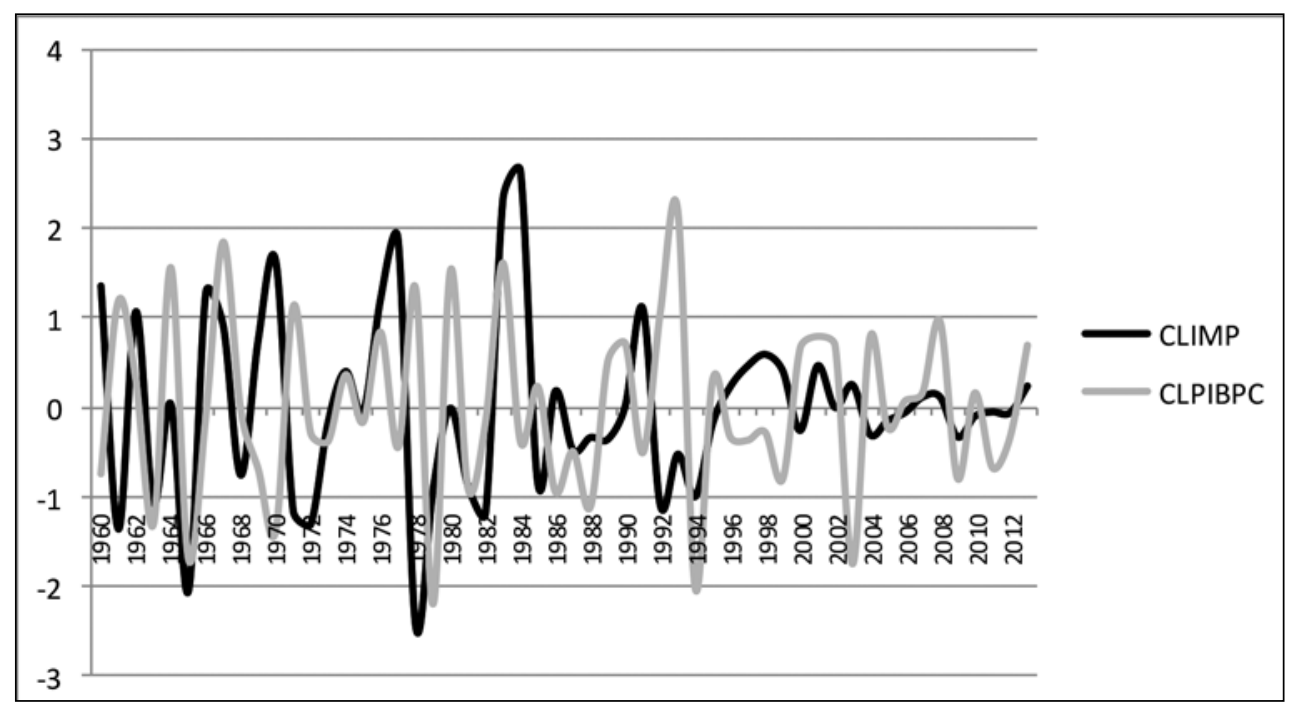

Fuente: Elaboración propia.

CLIMP es el ciclo en logaritmo de las importaciones del Cauca y CLPIBC es el ciclo en logaritmo del PIB del Cauca. Por ser cifras normalizadas, el eje Y debe ser entendido como desviaciones estándar.

En lo que respecta al nuevo milenio, los mayores renglones de importación fueron maquinaria y equipo (32.64\%), sustancias químicas (28.13\%), papel y sus productos $(19.85 \%)$, productos agropecuarios (8.94\%), de los cuales el $42.6 \%$ provinieron de Estados Unidos. Ya para 2005, las importaciones se concentraron en sustancias químicas, papel y maquinaria, donde Venezuela tuvo un alto grado de participación como proveedor. En años subsiguientes las importaciones se basaron en la compra de productos de papel $(23.1 \%$ del total) donde ahora fue México uno de los mejores socios comerciales. 
Finalizando la primera década del milenio, el 70\% de las compras se concentraron en las industrias de sustancias y productos químicos, metalúrgica, maquinaria y equipo y plástico-caucho, productos cuya procedencia era Estados Unidos, Venezuela y Corea, fundamentalmente.

En términos coyunturales, para 2014 las importaciones del Cauca ascendieron a US\$ 385.020.307 CIF, un $11 \%$ más que el año anterior. Dicha alza obedeció principalmente por el crecimiento de macro sectores como la manufactura e insumos (16\%) agroindustria (15\%) y servicios (11\%). Dichas im- portaciones están compuestas casi en su totalidad por los macro sectores no tradicionales $(99.9 \%)$ y el restante $0.1 \%$ por el sector tradicional (petróleo y sus derivados). Frente al comportamiento de las importaciones por macro sectores, de acuerdo al gráfico 3 se encuentra que las manufacturas e insumos participan con el $76 \%$ del total de las importaciones, seguido del sector servicios el cual alcanza una participación del $11 \%$, las prendas de vestir ocupan el tercer lugar con el $9 \%$ y la agroindustria el cuarto con $3,9 \%$ y por último el sector tradicional, con una participación del $0,1 \%$.

Gráfico 3. Porcentaje Importaciones por Macro sectores a precios CIF.

Miles de Millones de Pesos (100=2005). Cauca. 2013-2014

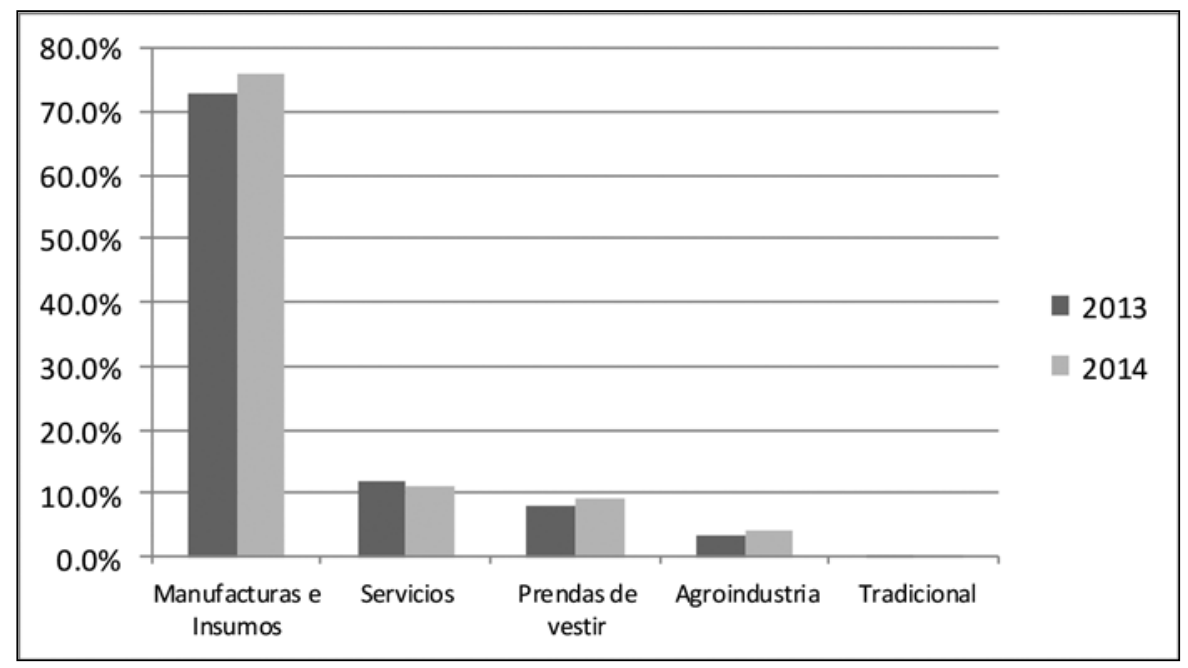

Fuente: Elaboración Cámara de Comercio del Cauca.

Dentro de los subsectores de las manufacturas, los de mejor comportamiento en 2014 fueron el plástico en formas primarias con una participación del $19 \%$ siendo el socio comercial mayoritario los Estados Unidos (17\%). En el caso de los subsectores de servicios el papel y cartones representan más de la mitad del total importado (52\%), los cuales fueron transferidos principalmente desde Estados Unidos (43\%). Dentro de prendas de vestir se destaca el subsector de textiles que ocupa el $94 \%$ del total de las compras. La procedencia de las importaciones de este macro sector, es China (22\%) y Estados Unidos (21\%) Finalmente, los principales subsectores de la agroindustria muestran que los cereales por ejemplo participan con el $43 \%$ del total de importaciones, seguido de las preparaciones alimenticias diversas $(23 \%)$, las cuales fundamentalmente provienen de nuevo de los Estados Unidos (49\%) y México (16\%).

En resumen, las importaciones del Cauca han tenido un crecimiento pronunciado a partir de la apertura económica a inicios de la década del noventa, y 
ha guardado históricamente en el periodo analizado algún grado de independencia del ciclo económico departamental, por lo menos de forma contemporánea, adicionalmente en la coyuntura de los últimos años importa principalmente manufacturas $e$ insumos, servicios, prendas de vestir y materiales para la agroindustria,siendo su principal socio comercial los Estados Unidos.

\section{Modelo Econométrico}

Con el fin establecer la relación entre el PIB del Cauca se plantea un modelo de demanda de importaciones basados en Khan (1975). Para esta investigación se supone que las importaciones dependen de los términos de intercambio y del PIB per cápita real del departamento, es decir siguiendo una demanda marshalliana típica, depende de los precios relativos y del ingreso, intentándo capturar la causalidad del PIB per cápita hacia las importaciones. Adicionalmente, siguiendo a Alonso \& Patiño (2007), se introduce en ella el PIB per cápita na- cional como una variable de control, lo cual permite intervenir los incrementos en la producción departamental generados por incrementos en la producción nacional (choques externos). Finalmente, de acuerdo a Jin (2002) como se desconoce ex ante si las importaciones de periodos pasados explican el comportamiento del PIB hoy o viceversa, se debe introducir un conjunto de rezagos. Así, la demanda de Khan se le denominará ahora demanda ampliada por importaciones de Khan.

De otro lado, para capturar la causalidad de las importaciones hacia el PIB, la demanda de importaciones ampliada debe plantearse como un modelo VAR, donde cada una de las variables endógenas ahora serán exógenas y deben estar en función de las restantes variables. Es decir, se crea un sistema que tiene tantas ecuaciones como variables exógenas y endógenas tenga la modelación inicial. En este orden de ideas, el modelo VAR (p) estándar, es el siguiente:

$$
\begin{aligned}
& \operatorname{LIMP}_{t}=\alpha+\sum_{i=1}^{k} \beta_{i} L I M P_{t-i}+\sum_{i=1}^{k} \gamma_{i} \operatorname{LPIBPN}_{t-i}+\sum_{i=1}^{k} \delta_{i} L P I B P C_{t-i}+\sum_{i=1}^{k} \epsilon_{i} L_{T C R_{t-i}}+\varepsilon_{1 t} \\
& \operatorname{LPIBPN}_{t}=\theta+\sum_{i=1}^{k} \vartheta_{i} \operatorname{LIMP}_{t-i}+\sum_{i=1}^{k} \mu_{i} \operatorname{LPIBN}_{t-i}+\sum_{i=1}^{k} \pi_{i} L P I B C_{t-i}+\sum_{i=1}^{k} \rho_{i} L T C R_{t-i}+\varepsilon_{2 t} \\
& L_{P I B P C}=\sigma+\sum_{i=1}^{k} \tau_{i} L_{t} I M P_{t-i}+\sum_{i=1}^{k} \varphi_{i} \operatorname{LPIBN}_{t-i}+\sum_{i=1}^{k} \omega_{i} L P I B C_{t-i}+\sum_{i=1}^{k} \zeta_{i} L T_{C} R_{t-i}+\varepsilon_{3 t} \\
& \operatorname{LTCR}_{t}=\psi+\sum_{i=1}^{k} \varsigma_{i} L I M P_{t-i}+\sum_{i=1}^{k} \varrho_{i} L_{P I B N}+i+\sum_{i=1}^{k} \varpi_{i} L P I B C_{t-i}+\sum_{i=1}^{k} v_{i} L T C R_{t-i}+\varepsilon_{4 t}
\end{aligned}
$$

Donde LIMP son las importaciones del Cauca en logaritmo, PIBPC Y PIBPN son el PIB departamental y nacional per cápita respectivamente expresados en logaritmo, y TCR recoge el tipo de cambio real peso-dólar, también en logaritmos, esta variable representa los términos de intercambio. Finalmente son los términos aleatorios de error de cada ecuación, los cuales se supone tienen media cero, varianza constante y covarianza nula. Los rezagos se denotan por la letra i, y el máximo rezago por la letra $\mathrm{k}$. El rezago máximo se determina a través de los criterios de información de tradicionales: Akaike y Schuwarz. Cabe anotar que por ser modelos doble logarítmicos, todos los parámetros del sistema representan elasticidades.

Si los resultados muestran a los parámetros en la ecuación 1 siendo no significativos y en la ecuación (3) siendo significativos se apoyará la idea alrededor de la incidencia de las importaciones de hoy 
en el PIB per cápita del futuro, ceteris paribus, y si además se muestran signos positivos indicarían un impacto creciente en el PIB per cápita. Lo anterior apoyaría los planteamientos de los autores disidentes del modelo de sustitución de importaciones; pero si los signos son negativos por vía contraria se respaldaría la idea de Prebisch (1950), por ejemplo. De otro lado, si los resultados encuentran a los parámetros en la ecuación 1 siendo significativos y en la ecuación (3) no siéndolos, mostraría la incidencia del PIB per cápita actual en las importaciones futuras, dándole la razón a Kahn (1975). Además, $y$ en concordancia con la elasticidad ingreso de las importaciones, si los signos son positivos indicarian una relación directa, clasificando los bienes importados como normales; y al contrario si son negativos la relación los clasificaría como inferiores, dejando otras cosas constantes.

Los parámetros asociados al PIB de Colombia operarían en concordancia con los asociados al PIB del Cauca, pero debido al comportamiento de la economía caucana, y su representación de tan solo el $1.7 \%$ en promedio de la economía nacional en los últimos 50 años, se espera un comportamiento no estadísticamente significativo de dichos parámetros. En el caso del tipo de cambio real, se espera un signo negativo de la elasticidad precio de la demanda, pues si el peso colombiano se devalúa, las importaciones caerán, pero por vía contraria una revaluación impulsaría las importaciones, ceteris paribus.

Para no estimar relaciones irrelevantes en el modelo VAR y con el fin de implementar funciones impulso-respuesta (las cuales ayudan a corroborar si el modelo está identificado además de ser coherente con el análisis teórico), se introducen restricciones transformando el modelo VAR en un SVAR. Para esto se requiere identificar las innovaciones (shocks) teniendo en cuenta la correlación entre los errores del modelo (Gómez \& Hernández, 2011). La ventaja con la utilización de los SVAR frente a los VAR es que tanto las funciones de impulso respuesta como las descomposiciones de varianza, pueden tener interpretaciones estructurales (Melo \& Hamann, 1999).
En este orden de ideas, siguiendo la metodología de Amisano \& Giannini (1996), el modelo SVAR es el siguiente:

$$
\boldsymbol{A}\left[\begin{array}{c}
\text { LIMP } \\
L P I B P N \\
L P I B P C \\
L T C R
\end{array}\right]=\boldsymbol{C}(\boldsymbol{L})\left[\begin{array}{c}
\operatorname{LIMP}_{t-1} \\
\operatorname{LPIBPN}_{t-1} \\
\operatorname{LPIBPC}_{t-1} \\
\operatorname{LTCR}_{t-1}
\end{array}\right]+\boldsymbol{B}\left[\begin{array}{c}
\varepsilon_{1 t} \\
\varepsilon_{2 t} \\
\varepsilon_{3 t} \\
\varepsilon_{4 t}
\end{array}\right]
$$

Donde A es una matriz simétrica de relaciones contemporáneas y $\mathbf{C}(\mathbf{L})$ una matriz simétrica de orden finito que captura la información para los distintos rezagos del modelo. La matriz $\mathbf{B}$ captura los respectivos mecanismos de transmisión de propagación del vector de términos de innovación los cuales se interpretan como choques no anticipados en el modelo. De forma reducida, se tiene que:

$$
Y=A^{-1} C(L) X+V
$$

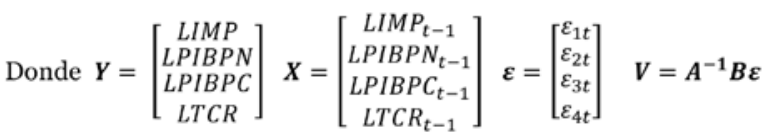

Las restricciones para la matriz A son las siguientes: un primer choque que impacta en el nivel de las importaciones del departamento del Cauca afecta negativamente al PIB caucano y nacional, toda vez que este rubro hace parte de la balanza comercial, pero no incide en el tipo de cambio ya son los precios de la moneda extranjera la señal para importar. El segundo choque que afecta al PIB per cápita nacional, incide únicamente en el PIB per cápita del Cauca, pues el segundo es una resultante o está contenido dentro del primero. El tercer choque, que se define como un impacto en el PIB per cápita del Cauca, se espera que no afecte al PIB per cápita nacional ya que su participación en los últimos años ha sido un poco superior al $1 \%$; y tampoco que tenga incidencia sobre el tipo de cambio real pues son esta depende de los precios nacionales e internacionales. El ultimo choque incidente en el tipo de cambio presenta un vector de no nulidad, ya que esta innovación impacta al resto de variables consideradas, puesto que las importaciones siguen la señal de los precios de la divisa, y las transacciones internacionales realizadas en dólares influyen en la balanza de pagos nacional y departamental. 
Debido a que el vector de innovaciones se modela como un sistema independiente de ecuaciones lineales la matriz $\mathbf{B}$ es una matriz idéntica. En otras palabras, el modelo SVAR es del tipo AB. Así, las restricciones de las matrices $\mathbf{A}$ y $\mathbf{B}$, son las siguientes:

$$
\boldsymbol{A}=\left[\begin{array}{cccc}
1 & 0 & 0 & a_{14} \\
a_{21} & 1 & 0 & a_{24} \\
a_{31} & a_{32} & 1 & a_{34} \\
0 & 0 & 0 & 1
\end{array}\right] \quad \boldsymbol{B}=\left[\begin{array}{llll}
1 & 0 & 0 & 0 \\
0 & 1 & 0 & 0 \\
0 & 0 & 1 & 0 \\
0 & 0 & 0 & 1
\end{array}\right]
$$

De acuerdo a Melo \& Hamann (1999) los pasos para la construcción del modelo SVAR son la estimación del modelo VAR estándar, luego se impone un número suficiente de restricciones sobre las matrices $\mathbf{A}$ y $\mathbf{B}$ con lo que se permita identificar los parámetros estructurales y obtener después las funciones impulso-respuesta.

Finalmente, frente a la minería de datos cabe resaltar que las series reales de las importaciones, PIB Cauca y Nacional fueron obtenidas del Sistema de Información Tecnoeconómica para el Cauca (SITEC) para el periodo 1960-1993 y para el periodo comprendido entre 1994-2013 por intermedio del Departamento Administrativo Nacional de Estadística (DANE); todas ellas con periodicidad anual y empalmadas a precios constantes de 2005, y el tipo de cambio real fue obtenido del Banco de la República. Así las cosas, las series analizadas tienen una extensión de 54 años.

\subsection{Integración y Cointegración}

Para conocer el grado de integración de las series analizadas se debe realizar la prueba de raíces unitarias, para lo cual se implementaron las pruebas de Dickey-Fuller Aumentada (ADF) y la prueba de Kwiatkowski-Phillips-Schmidt-Shin (KPSS). Dichas pruebas permiten identificar si existen raíces unitarias, es decir decide si la serie es o no estacionaria. La siguiente Tabla muestra los resultados: ${ }^{2}$
Tabla 2. Prueba de raíces Unitarias ADF y KPSS. Series en Logaritmos.

\begin{tabular}{|l|c|c|c|c|}
\hline & \multicolumn{2}{|c|}{ Niveles } & \multicolumn{2}{c|}{ Primeras Diferencias } \\
\hline Variable & ADF & KPSS & ADF & \multicolumn{1}{c|}{ KPSS } \\
\hline LIMP & -1.4503 & 0.8485 & $(-9.9901)^{\star \star \star}$ & $(0.1257)^{\star \star \star}$ \\
\hline LPIBPC & 2.2331 & 0.7967 & $(-3.68251)^{\star \star \star}$ & $(0.59166)^{\star \star}$ \\
\hline LPIBPN & -0.2916 & 0.8821 & $(-4.2899)^{\star \star \star}$ & $(0.88210)^{\star \star}$ \\
\hline LTCR & -2.1993 & 0.5370 & $(-4.5296)^{\star \star}$ & $(0.1097)^{\star \star \star}$ \\
\hline
\end{tabular}

Fuente: Elaboración propia con datos DANE y SITEC.

LIMP: logaritmo de las importaciones, LPIBPC: logaritmo del PIB per cápita Cauca, LPIBN: logaritmo del PIB per cápita nacional, LTCR: logaritmo de la Tasa de Cambio Real. ( $\left.{ }^{* * *}\right)$ representativo al $1 \%,\left(^{* *}\right)$ representativo al $5 \%,\left(^{*}\right)$ representativo al $10 \%$.

La tabla muestra un comportamiento no estacionario en niveles de todas las variables incluidas en el modelo, pero en primeras diferencias son integradas de orden 1 , es decir I (1), ya que en primeras diferencias son estadísticamente significativas de acuerdo a su p-valor. Por lo tanto, los tres primeros momentos de cada una de las variables aleatorias consideradas, son independientes del tiempo. A continuación, para indagar si la relación de las variables consideradas en el largo plazo no es falsa o espuria, es decir si las series están cointegradas, se aplica la prueba de Jhoansen (1988). Si dos o más variables están integradas en diferentes órdenes no habrá cointegración. Los resultados se muestran a continuación: ${ }^{3}$

Tabla 3. Test de Cointegración de Jhoansen.

\begin{tabular}{|l|l|l|l|}
\hline \multicolumn{1}{|c|}{ Vectores } & Valor porpio & \multicolumn{1}{c|}{ Traza } & \multicolumn{1}{c|}{ p-valor } \\
\hline Ninguno & 0.35 & 59.76 & 0.01 \\
\hline Al menos 1 & 0.33 & 38.17 & 0.02 \\
\hline Al menos 2 & 0.18 & 17.80 & 0.11 \\
\hline Al menos 3 & 0.14 & 7.56 & 0.10 \\
\hline
\end{tabular}

Fuente: Elaboración propia con datos DANE y SITEC. 
La prueba de Jhoansen arroja la existencia de al menos un vector de cointegración para la combinación de las series a largo plazo, pues el p-valor es igual al $5 \%$. Esto permite asegurar la validez del modelo VAR planteado anteriormente y la no generación de resultados falsos. Es decir, siguiendo a Greene (1999) las series por separado son aleatorias, pero de manera conjunta forman una combinación lineal estacionaria, permitiendo la solidez de su relación a largo plazo. De esta manera el modelo VAR se puede estimar sin ninguna dificultad estadística.

\subsection{Estimación del Modelo VAR}

Como en el apartado anterior las series son integradas de orden 1 y al menos existe un vector de cointegración. De otro lado, los criterios de información de Akaike y Scwartz encuentran que el número óptimo de rezagos es dos. En este orden de ideas, la estimación del modelo VAR (p) estándar de forma resumida es la siguiente: ${ }^{4}$

Tabla 4. Estimaciones Modelo VAR

\begin{tabular}{|c|c|c|c|c|}
\hline Variables & LIMP & LPIBPN & LPIBPC & LTCR \\
\hline \multirow{2}{*}{ C } & -1.058 & -0.333 & -0.687 & 2.735 \\
& {$[-0.11227]$} & {$[-1.81507]$} & {$[-1.47604]$} & {$[3.89326]$} \\
\hline \multirow{2}{*}{ LIMP(-1) } & 0.416 & -0.005 & 0.001 & 0.011 \\
& {$[2.84130]$} & {$[-1.77447]$} & {$[0.17559]$} & {$[1.03196]$} \\
\hline \multirow{2}{*}{ LIMP(-2) } & 0.094 & 0.005 & -0.002 & 0.014 \\
& {$[0.66565]$} & {$[1.98728]$} & {$[-0.33543]$} & {$[1.30135]$} \\
\hline \multirow{2}{*}{ LPIBPN(-1) } & -8.275 & 1.435 & -0.148 & -1.006 \\
& {$[-1.18753]$} & {$[10.5796]$} & {$[-0.43105]$} & {$[-1.93645]$} \\
\hline \multirow{2}{*}{ LPIBPN(-2) } & 9.053 & -0.450 & 0.159 & 1.058 \\
& {$[1.31225]$} & {$[-3.34744]$} & {$[0.46726]$} & {$[2.05703]$} \\
\hline \multirow{2}{*}{ LPIBPC(-1) } & 1.847 & -0.078 & 0.694 & -0.007 \\
& {$[2.24111]$} & {$[-1.33679]$} & {$[4.67525]$} & {$[-0.03268]$} \\
\hline \multirow{2}{*}{ LPIBPC(-2) } & 1.764 & 0.113 & 0.323 & -0.298 \\
& {$[1.96267]$} & {$[1.89136]$} & {$[2.13522]$} & {$[-1.30941]$} \\
\hline \multirow{2}{*}{ LTCR(-1) } & 1.190 & 0.031 & 0.075 & 1.010 \\
& {$[0.60580]$} & {$[0.81543]$} & {$[0.77446]$} & {$[6.89341]$} \\
\hline \multirow{2}{*}{ LTCR(-2) } & -2.064 & -0.002 & -0.006 & -0.162 \\
& {$[-1.02242]$} & {$[-0.06189]$} & {$[-0.06209]$} & {$[-1.07379]$} \\
\hline R2 & 0.855 & 0.9997 & 0.985 & 0.938 \\
\hline R2 Ajustado & 0.828 & 1.000 & 0.982 & 0.926 \\
\hline F estadístico & 31.6 & 18155.5 & 350.9 & 81.3 \\
\hline P Valof F & 0.000 & 0.000 & 0.000 & 0.000 \\
\hline
\end{tabular}

Fuente: con datos DANE y SITEC.

$\mathrm{El} P$ valor de la prueba $\mathrm{F}$ fue añadido por el autor

$4 \quad$ Los resultados ampliados se muestran en el anexo 3.
El sistema planteado muestra un buen ajuste a nivel global, donde el $\mathrm{R}^{2}$ supera al $85 \%$ en todos los modelos, y los p-valores de la prueba $\mathrm{F}$ son iguales a cero, y además el determinante de la matriz de covarianzas es igual a cero (ver anexo 3). Todo ello indica que el sistema consta de las variables indicadas para analizar el fenómeno de causalidad entre las importaciones y el PIB per cápita. Para evaluar la presencia de autocorrelación en los errores se efectuó la prueba de Portmanteau; y para evaluar la normalidad de los residuales se utilizó la prueba multivariada de errores aplicando la matriz de descomposición de Cholesky para la ortogonalización de la matriz de covarianzas. Finalmente, se evaluó la presencia de heterocedasticidad a través del test de White. Las tres pruebas tienen un p-valor por encima del $5 \%$, concluyendo la no existencia de autocorrelación ni heterocedasticidad y una distribución de errores multivariada normal. ${ }^{5}$

En términos específicos, el modelo inicial (ecuación 1), encuentra a las importaciones actuales del Cauca explicadas por su comportamiento del año anterior y por los comportamientos rezagados del PIB per cápita departamental hasta dos periodos. Las restantes variables no resultaron ser estadísticamente significativas, aunque siguiendo a Gómez \& Salazar (2014), encuentran que el tipo de cambio si influye en las importaciones, pero de manera contemporánea y no rezagada para el departamento del Cauca. Los resultados de los modelos 2 y 3 (ecuaciones 2 y 3), muestran una relación del PIB per cápita actual nacional y caucano, con su comportamiento pasado hasta dos periodos atrás, pero con las otras variables seleccionadas no, a través de evidencia estadística que así lo confirma. Este resultado no sorprende, pues el PIB per cápita de una nación o de un departamento depende básicamente de la demanda agregada. Finalmente, en el caso del modelo 4 (ecuación 4), el tipo de cambio contemporáneo depende de su comportamiento pasado y del PIB nacional hasta dos periodos atrás (las restantes variables resultan ser estadísticamente no significativas). Una causa posible es la mayor cantidad de ingresos de la nación

\footnotetext{
$5 \quad$ Ver anexo 4.
} 
hoy, elevando las compras de bienes extranjeros en el futuro, proceso coadyuvante en una mayor demanda de dólares.

Los resultados más importantes con la estimación del modelo se encuentran en las ecuaciones 1 y 3 ; ya que los parámetros en la ecuación 1 son significativos y en la ecuación (3) no lo son, por tanto es el PIB per cápita caucano actual quien incide en las importaciones futuras, o visto del otro lado, es el PIB per cápita hasta de dos periodos atrás quien incide en las importaciones actuales del departamento. La relación por tanto, no va desde las importaciones al PIB per cápita sino al contrario. Este hallazgo muestra que la hipótesis de Kahn (1975) es cierta y no la del modelo de sustitución de importaciones de Prebisch (1950) o la de sus disidentes.

Gráfico 4. Función Impulso-Respuesta. PIB Caucano.

\subsection{Funciones Impulso-Respuesta SVAR}

Para corroborar o reforzar los resultados anteriores, se implementó un análisis impulso-respuesta con restricciones. ${ }^{6}$ Este intenta evaluar la dinámica de los efectos que tienen choques exógenos sobre perturbaciones y por ende sobre el sistema. Si el intervalo de confianza mostrado por las líneas discontinuas contiene el valor de cero, las perturbaciones causan efectos estadísticamente nulos.

Los choques sobre la perturbación tienen efectos dinámicos diferentes sobre las importaciones y el PIB caucano. Como se muestra en el gráfico 4, en el primer caso es permanente, tomando entre $10 \mathrm{y}$ 12 periodos para estabilizarse en su nivel de largo plazo, es decir, un choque en el nivel de actividad económica tiene efectos contemporáneos y permanentes sobre la senda de las importaciones, los cuales se suavizan en el largo plazo.

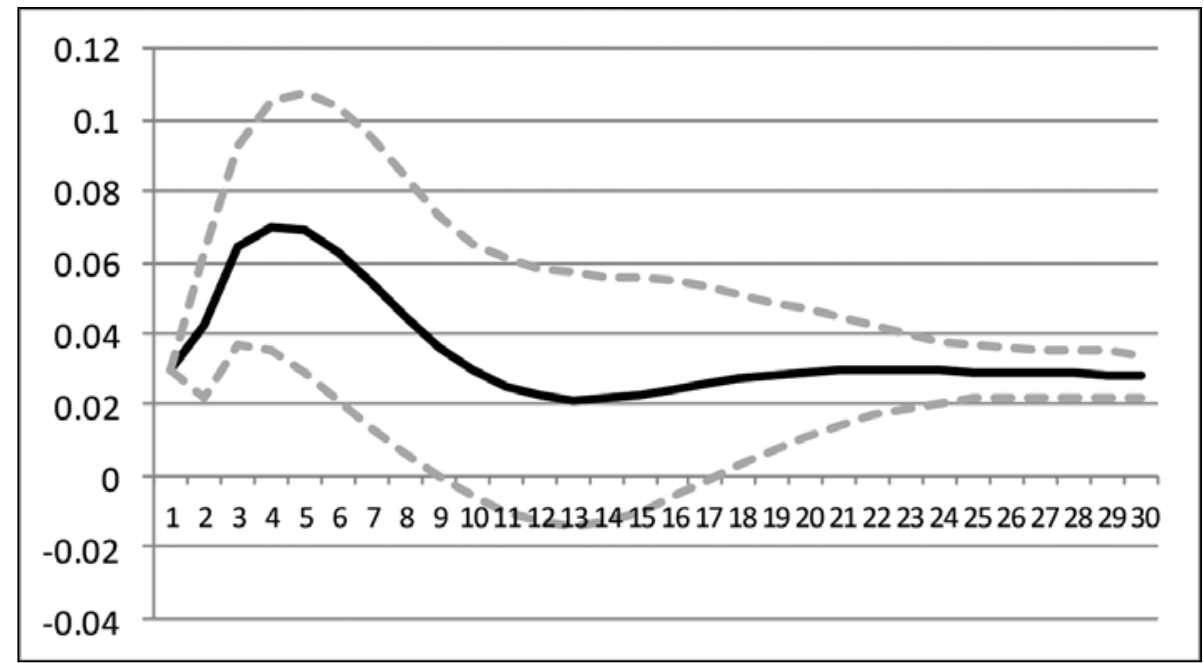

Fuente: Elaboración Propia con datos DANE y SITEC.

6 Los coeficientes estimados de las matrices A y B se muestran en el anexo 5 . 
De acuerdo al gráfico 4 se puede decir que ante un impulso en el PIB regional, las importaciones del Cauca responden aumentando, a partir del primer periodo. Este efecto se mantiene hasta el cuarto periodo, donde comienza a decaer hasta desaparecer el efecto en el doceavo período. Después de éste, las importaciones retornan a su equilibrio de largo plazo.
En el segundo caso, la función impulso respuesta del gráfico 5 muestra que el intervalo de confianza incluye el cero; por tanto los efectos sobre el PIB caucano de un choque en la perturbación, estadísticamente hablando, no son significativamente diferentes de cero.

Gráfico 5. Función Impulso-Respuesta. Importaciones.

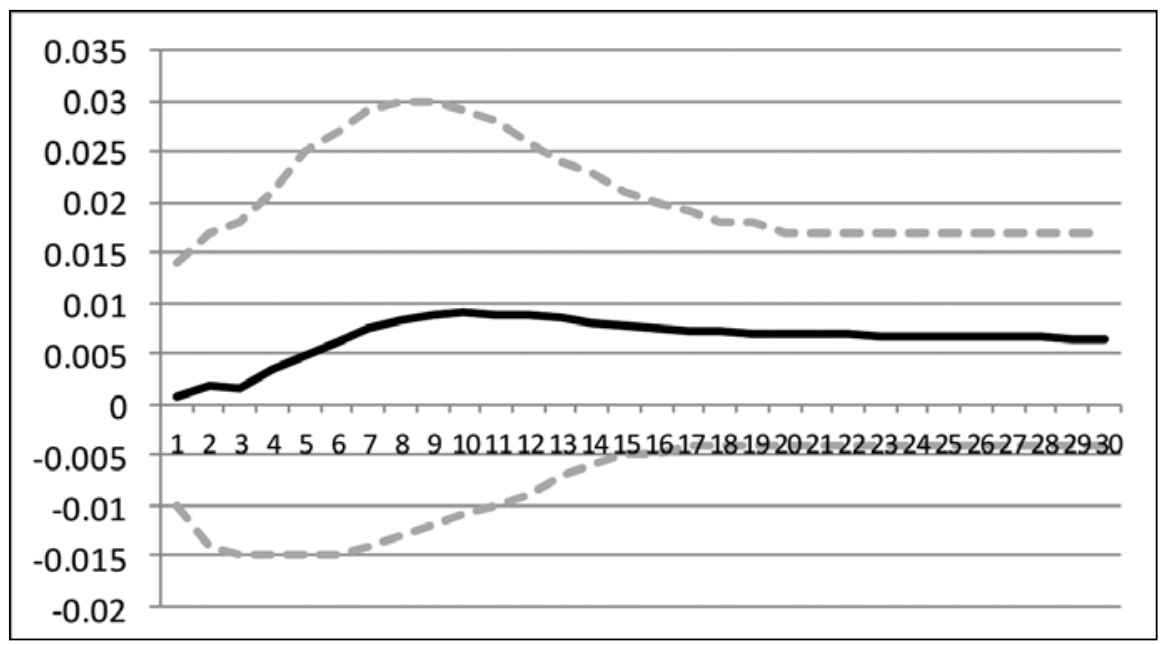

Fuente: Elaboración Propia con datos DANE y SITEC.

En otras palabras, el efecto de choques transitorios sobre las importaciones del departamento del Cauca no afecta al PIB en el largo plazo.

\section{Conclusiones}

La evidencia empírica muestra que en el Cauca se cumple la hipótesis Growth-driven Imports" o (GDI) y no la "Imports-led growth" (ILG), ya que la relación de causalidad entre el PIB per cápita y las importaciones en el Cauca va del primero hacia el segundo y no viceversa, resultado corroborado por el modelo VAR estándar y el SVAR. Es decir, las importaciones de este departamento dependen del comportamiento pasado de su PIB per cápita hasta en dos periodos atrás. Los signos positivos para los dos rezagos muestran además que el impacto del PIB per cápita hacia las importaciones es positivo y no negativo lo que apoya el modelo de demanda ampliada a la Khan (1975), en detrimento de la visión corto placista del comportamiento de las importaciones de Prebisch (1950) o la Ley de Thirlwall (1979), al igual que la idea largo placista de Griswold (2010) o Li, Greenaway \& Hine (2003).

Como la elasticidad ingreso es positiva se deduce que el Cauca importa bienes normales, aunque en el primer rezago es muy cercano a uno, es decir se importa en alguna medida bienes normales básicos necesarios, pero el segundo rezago muestra un valor lejano de la unidad, ósea se importa también bienes normales de lujo, los cuales posiblemente estén asociados a la importación de maquinaria y equipo para las empresas ubicadas en el norte del departamento. 
Una implicación importante de lo encontrado en el modelo es que el departamento del Cauca debe crecer para poder importar, y no importar para poder crecer. Por tal motivo no es de sorprender las cifras recientes del comportamiento de su economía, donde se muestra un mayor crecimiento tanto del PIB per cápita como de las importaciones. No hay cabida a paradojas, las importaciones reducen el PIB per cápita pero en el corto plazo de manera estática y no en el largo plazo de manera dinámica.

En cuanto a los tratados de libre comercio, el cumplimiento de la hipótesis de mayores importaciones vía crecimiento económico en el Cauca, implica que los acuerdos comerciales pueden jugar un papel complementario con mayores ingresos para importar antes que sustitutivo, es decir no es suficiente con firmar acuerdos comerciales con otros países que disminuyan los impuestos arancelarios por ejemplo, pues también se requiere de mayores niveles de ingreso. Siguiendo de nuevo a Gómez \& Salazar (2014), los acuerdos de libre comercio para el Cauca elevan los niveles de importaciones a largo plazo, pero hace falta decir que los ingresos también deben tenerse en cuenta.

Finalmente, en los modelos SVAR generalmente cuando se tienen muchos rezagos, se presentan elevados niveles de correlación en las variables explicativas, es decir, multicolinealidad. En este caso, como los rezagos tan solo son dos, la multicolinealidad es descartable. Además se dice que los modelos SVAR son generalmente ateóricos, pero para este caso están basados en la teoría de la demanda de importaciones Khan, el modelo de sustitución de importaciones de Prebisch y las restricciones teóricas introducidas.

\section{Referencias}

Abdulai, A. \& Jaquet, P. (2002). Exports and Growth: Cointegration and Causality Evidence for Côte d'Ivoire. Côte d'Ivoire: Blackwell Publishers.

Alonso, J. \& Patiño, C. (2007). ¿Crecer para Exportar o Exportar para Crecer? El caso del Valle del Cauca. Centro Regional de Estudios Económicos Cali. En: Ensayos Sobre Economía Regional. Banco de la República, 46: 1-28.

Amisano, G. \& Giannini, C. (1996). Topics in Structural VAR Econometrics. Second Edition, Springer. Disponible: http://
economia.unipv.it/eco-pol/Associazione\%20Giannini/Scritti/ svar_ag_1996.pdf

Asseery, A. \& Perdikis, N. (1993). The Functional Form of the Aggregate Import Demand Function: The Case of the GCC Countries. En: The Middle East Business and Economic Review, 5 (1): 34-38.

Awokuse, T. (2003): Is the Export-led Growth Hypothesis Valid for Canada?. En: Canadian Journal of Economics, 36, (1): 126-136.

Bahmani-Oskoee, M., \& Gobinda, G. (2004). Exchange Rate Sensitivity Of Japan`s Bilateral Trade Flow. En: Japan and the World Economy, 16 (1): 1-15.

Balaguer, J. \& Cantavella-Jordá, M. (2001): Examining the Exportled Growth Hypothesis for Spain in the Last Century. En: Applied Economics Letters, 8: 681-685.

Balaguer, L. \& Cantavella-Jordá, M. (2002), Tourism As A LongRun Economic Growth Factor: The Spanish Case. En: Applied Economics, 34: 877-884.

Becker, G. (1964). Human Capital: A Theoretical And Empirical Analysis, With Special Reference To Education. New York: Columbia University Press for National Bureau of Economic Research.

Brida, J., Pereyra, J., Such, M. \& Pulina, M. Causality between tourism and long-term economic growth: a critical review of the econometric literature. Working Paper. En: Munich Personal RePEc Archive. Paper, 3733: 1-27.

Cámara de Comercio del Cauca. (2013). Perfil de Comercio Exterior del Cauca. 55 p.

Cardona, O., Wilches, G., García, X., Mansilla, E., Ramírez, F. \& Marulanda, M. (2004). "Estudio sobre desastres ocurridos en Colombia: Estimación de pérdidas y cuantificación de costos. Banco Mundial, Agencia Colombiana de Cooperación Internacional". Departamento Nacional de Planeación. Bogotá. Disponible en: http://www.desenredando.org/public/varios/2007/varios_omar/ERNDesastres_Colombia_LaRed.pdf

DANE. (2014). Cuentas Nacionales Departamentales. Departamento Administrativo Nacional de Estadística. Disponible en: https://www.dane.gov.co/index.php/estadisticas-por-tema/ cuentasnacionales/cuentas-nacionales-departamentales

Eaton, J \& Kortum, S. (1999). International Patenting and Technology Diffusion: Technology and Measurement. En: International Economic Riview, 40: 537-570.

Furtado, C. (1969). Desarrollo y estancamiento en América latina: un enfoque estructuralista. En: Desarrollo Económico, 6 (22): 1-37.

Gómez, J \& Hernández, J. (2011). Composición Cambiaria y Poder Adquisitivo de las Reservas Internacionales. En: Borradores de Economía, 654: 1-54.

Gómez, M; \& Salazar; F. (2014). Demanda de Importaciones en la Región Pacífico Colombiana: Una Perspectiva de Largo Plazo. En: Entramado, 10 (2): 94-113.

Greene, W. (1999). Econometric Analysis. New Jersey: Prentince Hall.

Griswold, D. (2010). "Importaciones en aumento: ¿Bendición o maldición para la economía?" Disponible: http://www. 


\section{CAUSALIDAD ENTRE LAS IMPORTACIONES Y EL CRECIMIENTO ECONÓMICO: EVIDENCIA EMPÍRICA \\ PARA EL DEPARTAMENTO DEL CAUCA (COLOMBIA)}

elcato.org/importaciones-en-aumento-bendicion-o-maldicionpara-la-economia

Jin, J.C. (2002). Exports and Growth: is the Export-led Growth Hypothesis valid for provincial economies?. En: Applied Economics Letters, 34: 63-76.

Johansen, S. (1988): Statistical Analysis of Cointegrating Vectors. En: Journal of Economic Dynamics and Control, 12: 231-254.

Kee, H; Nicita, A; \& Olarreaga, M. (2008). Import Demand Elasticities and Trade Distortions. En: Review of Economics and Statistics, 90 (4): 666-682.

Keller, W. (2002). Geographic Localisation of International Technology Diffusion. En: American Economic Riview, 92 (1): 120-142.

Keynes, J. (1936). Teoría General de la Ocupación, el Interés y el Dinero. México: Fondo de Cultura Económica.

Khan, M. (1975). The Structure And Behaviour Of Imports Of Venezuela. En: Review of Economic and Statistics, 57: 221-224.

Khan, M. \& Ross, K. (1977). The Functional Form Of The Aggregate Import Equation. En: Journal of International Economics, 7: 149-160.

Krugman, P. \& Obstfeld, M. (2006). Economía Internacional: Teoría y Política. Madrid: Pearson.

Li, X; Greenway, D. \& Hane, R. (2003). "Imports of Services and Economic Growth: A Dynamic Panel Approach". Working Paper. GEP, Schooll of Economics, University of Nottingham. Disponible: https://www.coleurope.eu/content/development/ references-servicesEUinst/pdf/SETI/Imports\%20of\%20services\%20and\%20economic\%20growth\%20\%20-\%20a\%20 dynamic\%20panel\%20approach.PDF

Markusen, J. \& Rutherford, T. (2002). Developing Domestic Entrepreneurship and Growth through Imported Expertise. Working Paper. NBER, 7700: 1-30.

Maudos, J; Pastor, J \& Serrano, L. (1998). Convergencia en las regiones españolas: cambio técnico, eficiencia y productividad. En: Revista Española de Economía, Vol. 15, n² 2 , 1998 235-264
Mincer, J. (1974). Schooling, Experience, And Earnings. New York: Columbia University Press.

Melo, L. \& Hamann, F. (1999). "Inflación Básica, una estimación basada en modelos VAR estructurales". Monetaria CEMLA, 093: 1-28. Disponible: http://www.banrep.gov.co/sites/ default/files/publicaciones/pdfs/borra093.pdf

Metwally, M., \& Abdel-Rahman, M. (1985). Determination of Aggregate Expenditure of the Member States of the Gulf Cooperation Council. En: Asian Economic Review, 28 (1): p.36-52.

Nowak, J.,Sahli, M, \& Cortés, I. (2007). Tourism, Capital Good Imports and Economic Growth: Theory and Evidence for Spain. En: Tourism Economics, 13 (4): 515-536.

Panas, E. \& Vamvoukas, G. (2002): Further Evidence on the Export-led Growth Hypothesis. En: Applied Economics Letters, 9: 731-735.

Pinto, A. (1970). Naturaleza e implicaciones de la "heterogeneidad estructural" de la América Latina. En: El Trimestre Económico, 37 (1):83-100.

Prebisch, R. (1950). Teoría dinámica económica con particular aplicación a los países de América Latina. En: Obras. CEPAL, 21: 1-45.

Ramales, M. (2008): Industrialización por Sustitución de Importaciones (1940-1982) y modelo "secundario-exportador" (1983-2006) en perspectiva comparada. Málaga: Editorial Universidad de Málaga.

Ricardo, D. (1973). Principios de Economía Política y Tributación. México: Fondo de Cultura Económica.

Sharma, A. \& Panagiotidis, T. (2005). An Analysis of Exports and Growth in India: Cointegration and Causality Evidence (1971-2001). En: Review of Development Economics, 9 (2): 232-248.

Smith, A. (1987). Investigación Sobre la Naturaleza y Causas de la Riqueza de las Naciones. Barcelona: Editorial Oikos Tau.

Sunkel, O \& Paz, P. (1970). Subdesarrollo Latinoamericano y la Teoría del Desarrollo. México, D.F: Siglo Veintiuno.

Thirlwall, A. (1979). The balance of payments constraint as an explanation of international growth rate differences. Banca Nazionale Del Lavoro Quarterly, 32 (128): 45-53. 


\section{Anexos}

Anexo 1. Prueba ADF y KPSS

\begin{tabular}{|c|c|c|}
\hline \multicolumn{2}{|c|}{ Null Hypothesis: D(LIMP) has a unit root } & \\
\hline Exogenous: Constant & & \\
\hline \multicolumn{3}{|c|}{ Lag Length: 0 (Automatic - based on SIC, maxlag=10) } \\
\hline & t-Statistic & Prob.* \\
\hline Augmented Dickey-Fuller test statistic & -9.99012852 & 7.62E-11 \\
\hline Test critical \ $1 \%$ level & -3.56266941 & \\
\hline $5 \%$ level & -2.91877795 & \\
\hline $10 \%$ level & -2.59728515 & \\
\hline
\end{tabular}

\begin{tabular}{|c|c|c|}
\hline \multicolumn{2}{|c|}{ Null Hypothesis: D(LPIBPC) has a unit root } & \\
\hline Exogenous: Constant & & \\
\hline \multicolumn{3}{|c|}{ Lag Length: 1 (Automatic - based on SIC, maxlag=10) } \\
\hline & t-Statistic & Prob.* $^{*}$ \\
\hline Augmented Dickey-Fuller test statistic & -3.68250401 & 0.00726571 \\
\hline Test critical $1 \%$ level & -3.5654301 & \\
\hline $5 \%$ level & -2.91995181 & \\
\hline $10 \%$ level & -2.59790513 & \\
\hline
\end{tabular}

\begin{tabular}{|c|c|c|}
\hline \multicolumn{2}{|c|}{ Null Hypothesis: D(LPIBPN) has a unit root } & \\
\hline Exogenous: Constant & & \\
\hline \multicolumn{3}{|c|}{ Lag Length: 0 (Automatic - based on SIC, maxlag $=10$ ) } \\
\hline & t-Statistic & Prob.* \\
\hline Augmented Dickey-Fuller test statistic & -4.28990208 & 0.00122253 \\
\hline Test critical । $1 \%$ level & -3.56266941 & \\
\hline $5 \%$ level & -2.91877795 & \\
\hline $10 \%$ level & -2.59728515 & \\
\hline
\end{tabular}

\begin{tabular}{|c|c|c|}
\hline \multicolumn{2}{|c|}{ Null Hypothesis: $\mathrm{D}(\mathrm{LTCR})$ has a unit root } & \\
\hline Exogenous: Constant & & \\
\hline \multicolumn{3}{|c|}{ Lag Length: 0 (Automatic - based on SIC, maxlag=10) } \\
\hline & t-Statistic & Prob. $^{*}$ \\
\hline Augmented Dickey-Fuller test statistic & $=-4.52955784$ & 0.00058136 \\
\hline Test critical 、 $1 \%$ level & -3.56266941 & \\
\hline $5 \%$ level & -2.91877795 & \\
\hline $10 \%$ level & -2.59728515 & \\
\hline
\end{tabular}

\begin{tabular}{|l|l|r|}
\hline \multicolumn{2}{|l|}{ Null Hypothesis: D(LIMP) is stationary } \\
\hline \multicolumn{2}{|l|}{ Exogenous: Constant } \\
\hline \multicolumn{2}{|l|}{ Bandwidth: 4 (Newey-West automatic) using Bartlett kernel } \\
\hline \multicolumn{2}{|l|}{ Kwiatkowski-Phillips-Schmidt-Shin test statistic } & LM-Stat. \\
\hline Asymptotic critical values 1\% level & 0.1256586 \\
\hline & $5 \%$ level & 0.739 \\
\hline & $10 \%$ level & 0.463 \\
\hline
\end{tabular}

\begin{tabular}{|c|c|c|}
\hline \multicolumn{3}{|c|}{ Null Hypothesis: D(LPIBPC) is stationary } \\
\hline \multicolumn{2}{|c|}{ Exogenous: Constant } & \\
\hline \multicolumn{3}{|c|}{ Bandwidth: 4 (Newey-West automatic) using Bartlett kernel } \\
\hline & & LM-Stat. \\
\hline \multicolumn{2}{|c|}{ Kwiatkowski-Phillips-Schmidt-Shin test statistic } & 0.59166903 \\
\hline \multicolumn{2}{|c|}{ Asymptotic critical values $1 \%$ level } & 0.739 \\
\hline & $5 \%$ level & 0.463 \\
\hline & $10 \%$ level & 0.347 \\
\hline
\end{tabular}

\begin{tabular}{|c|c|c|}
\hline \multicolumn{3}{|c|}{ Null Hypothesis: D(LPIBPN) is stationary } \\
\hline \multicolumn{2}{|c|}{ Exogenous: Constant } & \\
\hline \multicolumn{3}{|c|}{ Bandwidth: 4 (Newey-West automatic) using Bartlett kernel } \\
\hline & & LM-Stat. \\
\hline \multicolumn{2}{|c|}{ Kwiatkowski-Phillips-Schmidt-Shin test statistic } & 0.09560238 \\
\hline \multicolumn{2}{|c|}{ Asymptotic critical values $1 \%$ level } & 0.739 \\
\hline & $5 \%$ level & 0.463 \\
\hline & $10 \%$ level & 0.347 \\
\hline
\end{tabular}

\begin{tabular}{|c|c|c|}
\hline \multicolumn{2}{|c|}{ Null Hypothesis: D(LTCR) is stationary } & \\
\hline \multicolumn{2}{|l|}{ Exogenous: Constant } & \\
\hline \multicolumn{3}{|c|}{ Bandwidth: 4 (Newey-West automatic) using Bartlett kernel } \\
\hline & & LM-Stat. \\
\hline \multicolumn{2}{|c|}{ Kwiatkowski-Phillips-Schmidt-Shin test statistic } & 0.1096588 \\
\hline \multicolumn{2}{|c|}{ Asymptotic critical values $1 \%$ level } & 0.739 \\
\hline & $5 \%$ level & 0.463 \\
\hline & $10 \%$ level & 0.347 \\
\hline
\end{tabular}

Fuente: Elaboración propia. 
Anexo 2. Test de Jhoansen

\begin{tabular}{|c|c|c|c|c|}
\hline \multicolumn{3}{|c|}{ Date: 12/02/16 Time: 09:31 } & & \\
\hline \multicolumn{5}{|c|}{ Sample (adjusted): 19632013} \\
\hline \multicolumn{5}{|c|}{ Included observations: 51 after adjustments } \\
\hline \multicolumn{5}{|c|}{ Trend assumption: No deterministic trend (restricted constant) } \\
\hline \multicolumn{5}{|c|}{ Series: LIMP LPIBPC LPIBPN LTCR } \\
\hline \multicolumn{5}{|c|}{ Lags interval (in first differences): 1 to 2} \\
\hline \multicolumn{5}{|c|}{ Unrestricted Cointegration Rank Test (Trace) } \\
\hline \multicolumn{2}{|c|}{ Hypothesized } & Trace & \multirow{2}{*}{\multicolumn{2}{|c|}{$\begin{array}{l}0.05 \\
\text { Critical Valut Prob.** }\end{array}$}} \\
\hline No. of $\mathrm{CE}(\mathrm{s})$ & Eigenvalue & Statistic & & \\
\hline None * & 0.34518732 & 59.7612553 & 54.0790364 & 0.01429775 \\
\hline At most $1^{*}$ & 0.3292889 & 38.1675455 & 35.1927546 & 0.0231857 \\
\hline At most 2 & 0.1818093 & 17.7972894 & 20.2618396 & 0.10547776 \\
\hline At most 3 & 1.38E-01 & 7.56363768 & 9.16454591 & 0.09969993 \\
\hline \multicolumn{5}{|c|}{ Trace test indicates 2 cointegrating eqn(s) at the 0.05 level } \\
\hline \multicolumn{5}{|c|}{${ }^{*}$ denotes rejection of the hypothesis at the 0.05 level } \\
\hline \multicolumn{5}{|c|}{ **MacKinnon-Haug-Michelis (1999) p-values } \\
\hline
\end{tabular}

Fuente: Elaboración propia. 
Anexo 3. Estimación Modelo VAR

\begin{tabular}{|c|c|c|c|c|c|}
\hline \multicolumn{5}{|c|}{ VAR Residual Portmanteau Tests for Autocorrelations } & \\
\hline \multicolumn{5}{|c|}{ Null Hypothesis: no residual autocorrelations up to lag $\mathrm{h}$} & \\
\hline \multicolumn{3}{|c|}{ Date: 12/02/16 Time:09:54 } & & & \\
\hline \multicolumn{2}{|c|}{ Sample: 19602013} & & & & \\
\hline \multicolumn{3}{|c|}{ Included observations: 52} & & & \\
\hline Lags & Q-Stat & Prob. & Adj Q-Stat & Prob. & df \\
\hline 1 & 1.82963748 & NA* & 1.86551272 & NA* & NA* \\
\hline 2 & 9.93565964 & NA* & 10.2957758 & NA* & NA* \\
\hline 3 & 16.8249937 & 0.95196616 & 17.6069058 & 0.93562063 & 28 \\
\hline
\end{tabular}

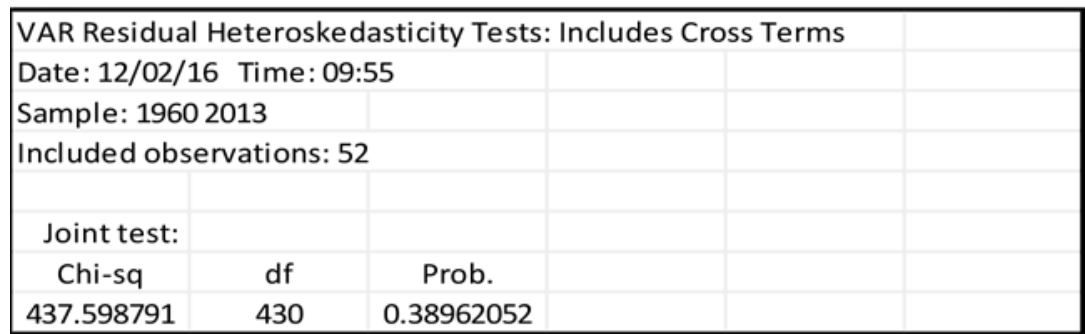

VAR Residual Normality Tests Orthogonalization: Cholesky (Lutkepohl) Null Hypothesis: residuals are multivariate normal Date: 12/02/16 Time: 09:57 Sample: 19602013 Included observations: 52 Component Skewness Chi-sq of

\begin{tabular}{|r|r|r|r|r|}
\hline 1 & -0.30355925 & 0.79861788 & 1 & 0.37150692 \\
\hline 2 & -0.76134578 & 5.02361079 & 1 & 0.02500398 \\
\hline 3 & -0.73906941 & 4.73393777 & 1 & 0.0295731 \\
\hline 4 & -0.07561168 & 0.04954842 & 1 & 0.82385092 \\
\hline Joint & & & & \\
\hline
\end{tabular}

Fuente: Elaboración propia. 
Anexo 4. Prueba de Autocorrelación de Portmanteau, Heterocedasticidad de White y Normalidad por Ortogonalización a la Cholesky

\begin{tabular}{|c|c|c|c|c|c|}
\hline \multicolumn{5}{|c|}{ VAR Residual Portmanteau Tests for Autocorrelations } & \\
\hline \multicolumn{5}{|c|}{ Null Hypothesis: no residual autocorrelations up to lag h } & \\
\hline \multicolumn{3}{|c|}{ Date: 12/02/16 Time:09:54 } & & & \\
\hline \multicolumn{2}{|c|}{ Sample: 19602013} & & & & \\
\hline \multicolumn{3}{|c|}{ Included observations: 52} & & & \\
\hline Lags & Q-Stat & Prob. & Adj Q-Stat & Prob. & df \\
\hline 1 & 1.82963748 & NA* & 1.86551272 & NA* & NA* \\
\hline 2 & 9.93565964 & NA* & 10.2957758 & NA* & NA* \\
\hline 3 & 16.8249937 & 0.95196616 & 17.6069058 & 0.93562063 & 28 \\
\hline
\end{tabular}

VAR Residual Heteroskedasticity Tests: Includes Cross Terms

Date: 12/02/16 Time: 09:55

Sample: 19602013

Included observations: 52

\begin{tabular}{|c|c|c|c|c|}
\hline Joint test: & & & \\
\hline Chi-sq & df & Prob. & \\
\hline 437.598791 & 430 & 0.38962052 \\
\hline
\end{tabular}

\begin{tabular}{|c|c|c|c|c|}
\hline \multicolumn{3}{|c|}{ VAR Residual Normality Tests } & & \\
\hline \multicolumn{5}{|c|}{ Orthogonalization: Cholesky (Lutkepohl) } \\
\hline \multicolumn{5}{|c|}{ Null Hypothesis: residuals are multivariate normal } \\
\hline \multicolumn{5}{|c|}{ Date: $12 / 02 / 16$ Time: $09: 57$} \\
\hline \multicolumn{5}{|c|}{ Sample: 19602013} \\
\hline \multicolumn{5}{|c|}{ Included observations: 52} \\
\hline \multirow[t]{5}{*}{ Component } & Skewness & Chi-sq & df & Prob. \\
\hline & -0.30355925 & 0.79861788 & 1 & 0.37150692 \\
\hline & -0.76134578 & 5.02361079 & 1 & 0.02500398 \\
\hline & -0.73906941 & 4.73393777 & 1 & 0.0295731 \\
\hline & -0.07561168 & 0.04954842 & 1 & 0.82385092 \\
\hline Joint & & 10.6057149 & 4 & 0.03137153 \\
\hline & & & & \\
\hline
\end{tabular}

Fuente: Elaboración propia. 
Anexo 5. Estimación de las matrices A y B modelo SVAR

\begin{tabular}{|c|c|c|c|}
\hline \multicolumn{4}{|l|}{ Estimated A matrix: } \\
\hline 1 & 0 & 0 & -1.04750154 \\
$-2.38 \mathrm{E}-04$ & 1 & 0 & 0.04665787 \\
-0.00055528 & -0.4490882 & 1 & 0.01057068 \\
0 & 0 & 0 & 1 \\
\hline Estimated B matrix: & & \\
\hline 0.89002939 & 0 & 0 & 0 \\
0 & 0.01710417 & 0 & 0 \\
0 & 0 & 0.04339322 & 0 \\
0 & 0 & 0 & 0.06656096 \\
\hline
\end{tabular}

Fuente: Elaboración propia. 